\title{
Discovery of Fast X-ray Oscillations During the 1998 Giant Flare from SGR $1900+14$
}

\author{
Tod E. Strohmayer ${ }^{1}$ and Anna L. Watts ${ }^{2}$
}

\begin{abstract}
We report the discovery of complex high frequency variability during the August 27, 1998 giant flare from SGR 1900+14 using the Rossi X-ray Timing Explorer (RXTE). We detect an $\approx 84 \mathrm{~Hz}$ oscillation (QPO) during a $1 \mathrm{~s}$ interval beginning approximately $1 \mathrm{~min}$ after the initial hard spike. The amplitude is energy dependent, reaching a maximum of $26 \%$ (rms) for photons above $30 \mathrm{keV}$, and is not detected below $11 \mathrm{keV}$, with a $90 \%$ confidence upper limit of $14 \%$ (rms). Remarkably, additional QPOs are detected in the average power spectrum of data segments centered on the rotational phase at which the $84 \mathrm{~Hz}$ signal was detected. Two signals, at 53.5 and $155.1 \mathrm{~Hz}$, are strongly detected, while a third feature at $28 \mathrm{~Hz}$ is found with lower significance. These QPOs are not detected at other rotational phases. The phenomenology seen in the SGR $1900+14$ flare is similar to that of QPOs recently reported by Israel et al. (2005) from the December 27, 2004 flare from SGR 1806-20, suggesting they may have a common origin, perhaps torsional vibrations of the neutron star crust. Indeed, an association of the four frequencies (in increasing order) found in SGR 1900+14 with $l=2,4,7$, and 13 toroidal modes appears plausible. We discuss our findings in the context of this model and show that if the stars have similar masses then the magnetic field in SGR 1806-20 must be about twice as large as in SGR 1900+14, broadly consistent with estimates from pulse timing.
\end{abstract}

Subject headings: stars: magnetic-pulsar: individual (SGR 1900+14)--stars: neutron-stars: rotation-stars: oscillations-X-rays: stars

\section{Introduction}

The giant flares produced by highly magnetized neutron stars, "magnetars," are amongst the most energetic of stellar phenomena. Three such flares have been recorded since the

\footnotetext{
${ }^{1}$ Exploration of the Universe Div., NASA/GSFC, Greenbelt, MD 20771; stroh@milkyway.gsfc.nasa.gov

${ }^{2}$ Exploration of the Universe Div., NRC/GSFC, Greenbelt, MD 20771; anna@milkyway.gsfc.nasa.gov
} 
advent of satellite borne high energy detectors. The most recent event, from SGR 1806-20 (hereafter, 1806), was also the most luminous, generating upwards of $10^{46} \mathrm{erg}$ in a matter of minutes (Terasawa et al. 2005; Palmer et al. 2005). At its peak the prodigious photon flux paralyzed almost all satellite instruments which viewed the event. The magnetic instability which powers such flares is likely associated with large scale fracturing of the neutron star crust (Flowers \& Ruderman 1977; Thompson \& Duncan 1995; Duncan 1998; Thompson \& Duncan 2001; Schwartz et al. 2005), and will almost certainly excite large scale seismic motions within the star.

Recently, Israel et al. (2005) presented evidence from Rossi X-ray Timing Explorer (RXTE) observations of transient, high frequency quasi-periodic oscillations (QPOs) during the pulsating tail of the giant flare from 1806. They found 92.5 Hz QPOs approximately 200 s after the onset of the flare. These QPOs appear to be associated with a spectrally hard, unpulsed emission component, and are only detected during particular rotational phases. The presence of lower frequency, $\approx 18$ and $30 \mathrm{~Hz}$ QPO features was also claimed. Israel et al. (2005) suggested that these QPOs may be due to global oscillation modes of the neutron star. In particular, they propose an association with torsional (toroidal) vibrations of the neutron star crust (Schumaker \& Thorne 1983; McDermott, van Horn \& Hansen 1988; Strohmayer 1991; Duncan 1998). Toroidal oscillations can be readily excited by crust fracturing, as demonstrated by the devastating 2004 Sumatra-Andaman earthquake (Park et al. 2005), and have periods within the range observed. Coupling to the magnetosphere via the magnetic field threading the crust appears to be a plausible method of modulating the X-ray flux during the flare, but a detailed model of this process is needed. We note that Barat et al. (1983) suggested torsional vibrations might explain a claimed 23 ms modulation during the initial spike of the March 5, 1979 flare from SGR 0526-66.

Motivated by the work of Israel et al. (2005) we investigated the fast timing properties of the only other giant flare observed with high sensitivity instrumentation; the August 27, 1998 event from SGR 1900+14 (hereafter, 1900, Hurley et al. 1999). Feroci et al. (2001) carried out a detailed study of the BeppoSAX and Ulysses data from this flare. They found a clear signal of the neutron star's spin frequency $(\sim 0.2 \mathrm{~Hz})$ and its harmonics in power spectra of the event, and modelled the pulsating tail with a trapped fireball model. Guidorzi et al. (2004) investigated the flare's fast timing properties with BeppoSAX, but could not search the entire event due to a dearth of high time resolution data. Nevertheless, they presented evidence for a power law noise component extending above $100 \mathrm{~Hz}$.

In this Letter we report the results of a timing study of this flare using the Proportional Counter Array (PCA) onboard RXTE. We detect a transient QPO at $\approx 84 \mathrm{~Hz}$ during a $\sim 1$ $\mathrm{s}$ interval approximately 1 minute after flare onset, and a pair of persistent QPOs at 53.5 
and $155.5 \mathrm{~Hz}$ associated with the rotational phase of the $84 \mathrm{~Hz}$ signal. After describing our timing study we conclude with a discussion of our findings in the context of neutron star toroidal oscillation modes.

\section{Observations and Data Analysis}

During the August 27, 1998 flare from 1900, RXTE was pointed at the cataclysmic variable AM Her, about $42^{\circ}$ away from it. The PCA was configured in one of the "good xenon" data modes (Goodxenon16s) with $\approx 1 \mu$ s resolution. This mode was identical to one running at the time of the 1806 flare except for a longer read-out cycle (16 s compared with $2 \mathrm{~s}$ for 1806) that led to data gaps during the flare. Nevertheless, high time resolution, unsaturated data are available for portions of the pulsating tail. Fig. 1 shows the lightcurve of the flare from both the Standard1 (bottom) and Goodxenon16s (top) modes. The Standard1 mode produces $1 / 8$ s resolution light curves across the full PCA bandpass (nominally 2 - 90 $\mathrm{keV}$ ). The gaps in the Goodxenon16s data occur because the buffer is filled before it can be read out.

Because of the gaps we first searched each good data interval separately, starting with the first interval following the peak of the flare (severe deadtime at the peak precludes a meaningful search there). We calculated power spectra with a Nyquist frequency of 2048 $\mathrm{Hz}$ using the good data in each interval. Based on the results of Israel et al. (2005) we averaged the power spectra to $\approx 1 \mathrm{~Hz}$ frequency resolution to best match their reported QPO coherence values. We did not find any candidate detections in the first three data intervals, however, the power spectrum of the fourth interval (marked by the vertical dotted lines in Fig. 1) shows a significant peak at $\approx 84 \mathrm{~Hz}$. Fig. 2 shows the power spectrum from this interval in the $10-2048 \mathrm{~Hz}$ band at $1 \mathrm{~Hz}$ resolution. We estimated the significance using a $\chi^{2}$ distribution with 16 degrees of freedom, which is the distribution expected based on the number of independent frequency bins averaged. We fitted the observed distribution of noise powers with a $\chi^{2}$ function and confirmed that it is consistent with the expected noise distribution (see the inset to Fig. 2). The peak at $84 \mathrm{~Hz}$ has a single trial probability of $6.8 \times 10^{-10}$ based on this noise distribution. Multiplying by the number of frequency bins searched (2048) and a "confirmation bias" reflecting the fact that this was the fourth spectrum searched we arrive at a significance of $5.6 \times 10^{-6}$, a fairly robust detection. The peak power corresponds to an average amplitude (rms) of $10.2 \%$.

We next constructed a dynamic power spectrum using the $Z^{2}$ statistic (Strohmayer \& Markwardt 1999). Fig. 3 shows a contour plot of this spectrum and the light curve of the fourth data segment. The $84 \mathrm{~Hz}$ signal is clearly localized in time near the largest peak of the 
four-peaked profile that emerged during the pulsating tail (Hurley et al. 1999; Feroci et al. 2001). Although the signal is not centered on the peak, we cannot rule out some association with its right flank. This behavior is similar to that reported by Israel et al. (2005), in that the oscillations are not centered on a peak. To quantify the amplitude variation with energy we computed power spectra for different energy bands, restricting the time range to the interval in which the signal was detected. The amplitude measurements are corrected for background, using data prior to the flare as an estimate of the total background. The amplitude (rms) in the $12-90 \mathrm{keV}$ band is $20 \pm 3 \%$, increasing to $26 \pm 4 \%$ for photons above $30 \mathrm{keV}$. For photon energies less than $18 \mathrm{keV}$ we have a $90 \%$ confidence upper limit of about $14 \%$. We note that a detailed spectral study of the flare is complicated by the fact that 1900 was not in the PCA field of view.

Israel et al. (2005) found that the QPOs in 1806 had a dependence on rotational phase. To test for such behavior we computed an average power spectrum using $1.5 \mathrm{~s}$ intervals centered on and beginning at the rotational phase of the $84 \mathrm{~Hz}$ signal. For the spin period we used the results of Woods et al. (1999). The resulting power spectrum from 20 independent intervals is plotted in Fig. 4 (middle and upper curves), and clearly shows an additional pair of QPOs, and perhaps a third. The time interval of this spectrum extends about $90 \mathrm{~s}$ beyond the $84 \mathrm{~Hz}$ detection. We fit this spectrum with a power law continuum and a pair of Lorenztian profiles for the two strongest QPOs. This model fits well, with a $\chi^{2} /$ dof $=904 / 892$. The frequencies of the strongest QPOs are $53.5 \pm 0.5$ and $155.1 \pm 0.2$ $\mathrm{Hz}$, with coherence values $Q \equiv \nu / \sigma_{\nu}$ of 22 and 55 , respectively. Their average amplitudes (rms) are $6.7 \pm 1.5$ and $10.9 \pm 2.2 \%$. These QPOs are very significant; removal of the 53 and $155 \mathrm{~Hz}$ components increases $\chi^{2}$ by 72 and 134, respectively. The middle and top curves in Fig. 4 show the same power spectrum at different frequency resolution, 4 and $4 / 3 \mathrm{~Hz}$, respectively. At the higher frequency resolution (top) there is a suggestive peak at about 28 $\mathrm{Hz}$. Including a Lorentzian for this feature improves $\chi^{2}$ by 22.1 , which is a bit better than a $3 \sigma$ deviation, so we consider this a tentative detection. Finally, the strength of these features is a function of rotational phase. To confirm this we computed average power spectra in the same manner but centered on rotational phases away from that of the $84 \mathrm{~Hz}$ signal. Fig. 4 (bottom) shows the average power spectrum for phases centered $\pm 1.5 \mathrm{~s}$ from that of the 84 $\mathrm{Hz}$ signal. The QPOs are clearly not detected. This analysis also confirms that the detected signals cannot be detector artifacts, but must be intrinsic to the source. 


\section{Discussion}

Our results show striking similarities with those obtained for the 1806 flare by Israel et al. (2005). First, we find a frequency within $8 \%$ of the $92.5 \mathrm{~Hz}$ feature in 1806, suggesting the same underlying mechanism. Second, the strongest signal is transient, appears to be associated with a particular rotational phase and is not centered on a profile peak. Although the $92 \mathrm{~Hz}$ signal in 1806 persists for longer, this could be due to its larger overall luminosity, and we may have missed occurrences of the $84 \mathrm{~Hz}$ signal due to data gaps. In both flares the amplitude is greater at higher photon energies. However, there is no obvious hard DC component associated with the $84 \mathrm{~Hz}$ QPO in 1900, as in 1806 (Israel et al. 2005).

The firm detection of multiple QPOs associated with a specific rotational phase provides compelling evidence that the modulations are related to a particular region on the stellar surface, perhaps the site of a crust fracture, or a certain magnetic field bundle. Moreover, the comparisons to 1806 strongly suggest we are seeing a similar phenomenon in 1900 . Israel et al. (2005) conjectured that toroidal oscillations of the neutron star might explain the observed frequencies. We now explore the implications of our findings in the context of this model.

For a non-rotating, non-magnetic star, Duncan (1998) estimates the period of the fundamental $(l=2, n=0)$ toroidal mode (denoted $\left.{ }_{2} t_{0}\right)$ to be

$$
P\left({ }_{2} t_{0}\right)=33.6 R_{10} \frac{0.87+0.13 M_{1.4} R_{10}^{-2}}{\left(1.71-0.71 M_{1.4} R_{10}^{-1}\right)^{1 / 2}} \mathrm{~ms},
$$

where $R_{10}=R / 10 \mathrm{~km}$ and $M_{1.4}=M / 1.4 M_{\odot}$. The periods of higher order $n=0$ modes ( $n \geq 1$ modes have frequencies that are too high) are given by

$$
P\left({ }_{l} t_{0}\right)=P\left({ }_{2} t_{0}\right)\left(\frac{6}{l(l+1)}\right)^{1 / 2}\left[1+\left(\frac{B}{B_{\mu}}\right)^{2}\right]^{-1 / 2},
$$

where the factor in square brackets is a magnetic correction, $B_{\mu} \approx 4 \times 10^{15} \rho_{14}^{0.4} \mathrm{G}$, and $\rho_{14}$ is the crustal density in units of $10^{14} \mathrm{~g} \mathrm{~cm}^{-3}$. Duncan (1998) argues that $\rho_{14} \sim 1$ is appropriate since the mode periods are largely set by conditions in the deep crust. In deriving equation (2) it is assumed that magnetic tension boosts the crustal shear modulus isotropically. However, the field configuration and corresponding non-isotropic effects could alter the magnetic correction dramatically (Messios, Papadopoulos \& Stergioulas 2001).

Israel et al. (2005) suggest that their $92.5 \mathrm{~Hz}$ QPO, and a weaker feature at $30.4 \mathrm{~Hz}$ could be due to the ${ }_{7} t_{0}$ and ${ }_{2} t_{0}$ modes, respectively. Preferential excitement of higher $l$ modes is not unreasonable, being set by the fracture geometry: such an effect was seen in 
the spectrum of Earth modes excited by the 2004 Sumatra-Andaman earthquake (Park et al. 2005). With at least three frequencies in 1900 we can explore whether a sequence of modes of different $l$ is plausible. We find only one reasonably plausible sequence, that where the frequencies are due to the $l=4(53 \mathrm{~Hz}), l=7(84 \mathrm{~Hz})$ and $l=13(155 \mathrm{~Hz})$ modes (see Figure 4 ). With this sequence the inferred ${ }_{2} t_{0}$ mode frequency is $\approx 28 \mathrm{~Hz}$, lower than that for 1806, but, intriguingly, consistent with the frequency of our tentatively detected 28 $\mathrm{Hz}$ QPO. Although the mode frequencies in this sequence do not precisely match our QPO centroid frequencies, magnetic field corrections could account for the modest offsets.

The different inferred $l=2$ mode frequencies imply that the stellar parameters must differ. Given an equation of state (EOS), equations (1)-(2) specify the relationship between mass and magnetic field necessary to give an oscillation at a desired frequency. Fig. 5 shows, for several EOS, the stellar parameters that give ${ }_{2} t_{0}$ oscillations at $28 \mathrm{~Hz}(1900)$ and $30.4 \mathrm{~Hz}$ (1806). If the stars have similar magnetic field strengths, their masses must differ by more than $0.2 M_{\odot}$. This seems unlikely unless magnetar masses differ significantly from those of other non-accreting neutron stars; a study of radio pulsars by Thorsett \& Chakrabarty (1999) found the masses to be consistent with a narrow Gaussian distribution, $M=1.35 \pm 0.04 M_{\odot}$. More likely the stars have similar masses but different magnetic fields. If we assume that both stars have masses $\approx 1.35 M_{\odot}$, we can immediately rule out the softest EOS; and the hardest EOS predicts magnetic fields for both systems that are far larger than those inferred from timing studies (Woods et al. 2002). Neither the AP3 nor the AP4 EOS can be ruled out, given that the inferred $l=2$ mode frequency of $28 \mathrm{~Hz}$ has a $\pm 0.5 \mathrm{~Hz}$ uncertainty. However, the magnetic fields that one would infer from these EOS agree well with those derived from timing measurements of both stars, which suggests that 1806 has a field strength approximately double that of 1900 (Woods \& Thompson 2005).

Feroci et al. (2001) and Thompson \& Duncan (2001) have explored the radiative mechanisms associated with magnetar flares. They associate the four-peaked profile during the 1900 flare with X-ray "jets" produced by the strong polarization dependence of scattering in the $\approx 10^{15} \mathrm{G}$ field. These jets form close to the surface and likely reflect the multipole structure of the magnetic field. Our detection of QPO signals shortly after the emergence of the four-peaked profile, in association with one of these "jets", is consistent with a mechanism that produces motions at or near the stellar surface. Indeed, the presence of beamed emission offers a plausible scenario to achieve large amplitudes from modest surface displacements. 


\section{Summary}

The discovery of high frequency QPOs with the right $[l(l+1)]^{1 / 2}$ scaling during a second magnetar flare is strong evidence that we may be seeing the influence of global toroidal modes of neutron star crusts on the X-ray emission from these objects. The recent results suggest these QPOs may be ubiquitous, in which case future observations could provide a new probe of neutron star structure. Efforts should be made to see that sensitive instrumentation is in place to observe the next such flare.

\section{REFERENCES}

Barat, C. et al. 1983, A\&A, 126, 400

Duncan, R.C., 1998, ApJ, 498, L45

Feroci, M., et al., 2001, ApJ, 549, 1021

Guidorzi, C. et al., 2004, A\&A, 416, 297

Flowers, E., Ruderman, M.A., 1977, ApJ, 215, 302

Hurley, K., et al., 1999, Nature, 397, 41

Israel, G., et al., 2005, ApJ, 628, L53

Lattimer, J.M., Prakash, M., 2001, ApJ, 550, 426

McDermott, P.N., van Horn, H.M., Hansen, C.J., 1988, ApJ, 325, 725

Messios, N., Papadopoulos, D.B., Stergioulas, N., 2001, MNRAS, 328, 1161

Palmer, D.M., et al., 2005, Nature, 434, 1107

Park, J., et al., 2005, Science, 308, 1139

Schumaker, B.L., Thorne, K.S., 1983, MNRAS, 203, 457

Schwartz, S.J., et al., 2005, ApJ, 627, L129

Strohmayer, T.E., 1991, ApJ, 372, 573

Strohmayer, T.E., Markwardt, C.B., 1999, ApJ, 516, L81

Terasawa, T., et al., 2005, Nature, 434, 1110 
Thompson, C., \& Duncan, R. C. 2001, ApJ, 561, 980

Thompson, C., Duncan, R.C., 1995, MNRAS, 275, 255

Thorsett, S.E., Chakrabarty, D., 1999, ApJ, 512, 288

Woods, P.M., et al., 2002, ApJ, 576, 381

Woods, P.M. et al. 1999, ApJ, 524, L55

Woods, P.M., Thompson, C., to appear in Compact Stellar X-ray Sources, eds. W.H.G. Lewin and M. van der Klis, Cambridge University Press 


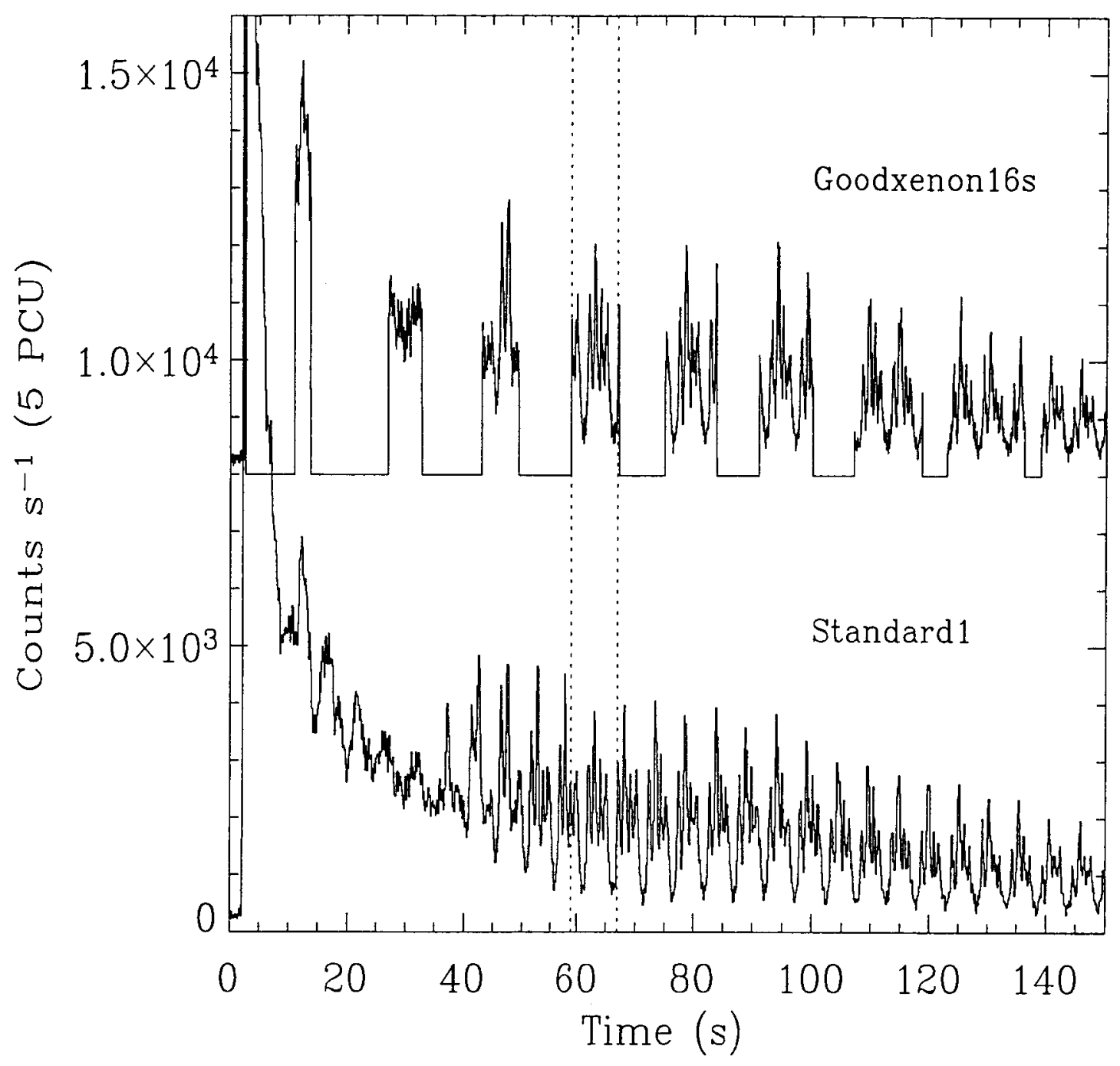

Fig. 1.- Light curves of the August 27, 1998 flare from 1900. The Goodxenon16s curve (top) is plotted at $1 / 16 \mathrm{~s}$ resolution, and is displaced vertically by $8,000 \mathrm{~s}^{-1}$ for clarity. The data gaps result from the finite buffer size and the long time between read outs (16 s). The Standard1 data (bottom) provides an uninterrupted view of the flare. The vertical dotted lines mark the time interval in which the $84 \mathrm{~Hz}$ signal was first detected. Time zero corresponds to MJD 51052.33088176 (TT). 


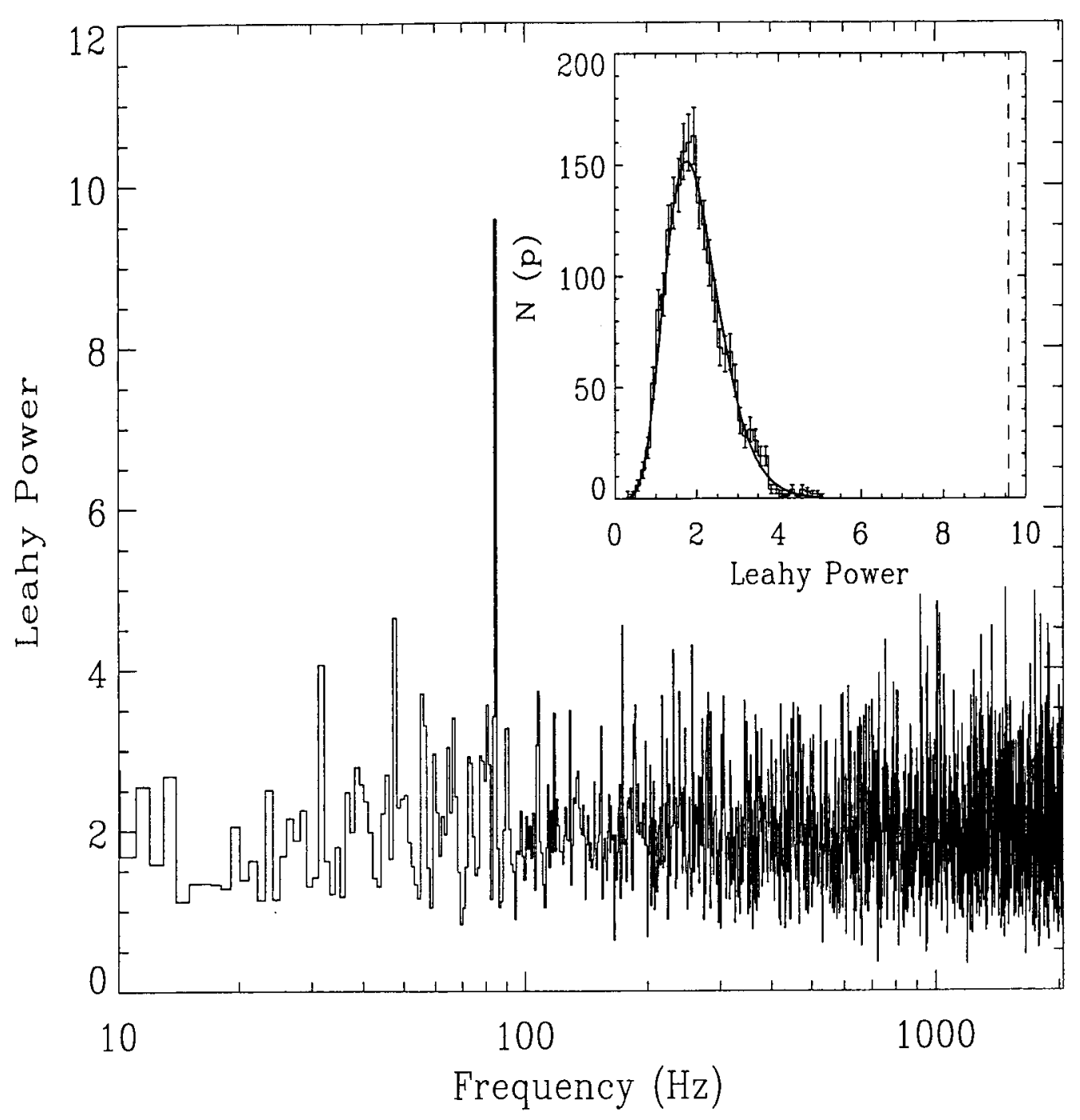

Fig. 2.- Power spectrum in the $10-2048 \mathrm{~Hz}$ band of the fourth good data interval from the Goodxenon16s data (see Fig. 1), showing the $84 \mathrm{~Hz}$ peak. The frequency resolution is 1 $\mathrm{Hz}$, and the Nyquist frequency is $2048 \mathrm{~Hz}$. The inset panel shows the distribution of powers as a histogram, along with the best fitting $\chi^{2}$ function (solid). The peak power in the $84 \mathrm{~Hz}$ feature is marked with the vertical dashed line. 


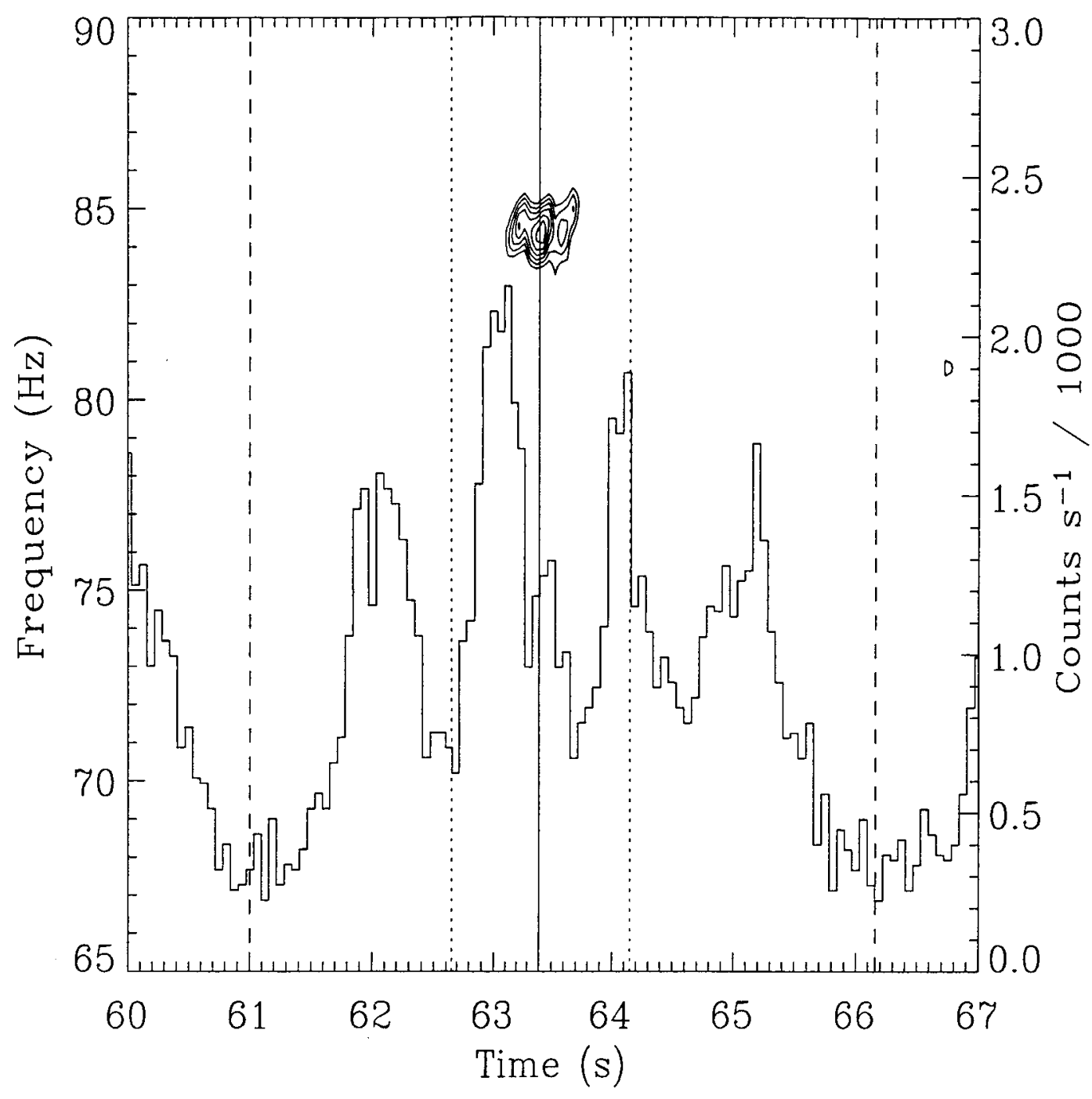

Fig. 3.- Dynamic power spectrum of the data interval containing the QPO. Spectral power contours were computed from $0.5 \mathrm{~s}$ segments with a new segment beginning every $0.1 \mathrm{~s}$. The lowest and highest contours correspond to single trial probabilities of $4.5 \times 10^{-5}$ and $1.7 \times 10^{-10}$, respectively. The signal is localized near the brightest peak of the four-peaked profile, but it is not centered on the peak. The vertical dotted lines denote the phase range used to compute average power spectra (see Fig. 4). The vertical solid line marks the center of this range. One rotational cycle $(5.16 \mathrm{~s}$ period) is marked by the vertical dashed lines. The time scale is the same as in Fig. 1. 


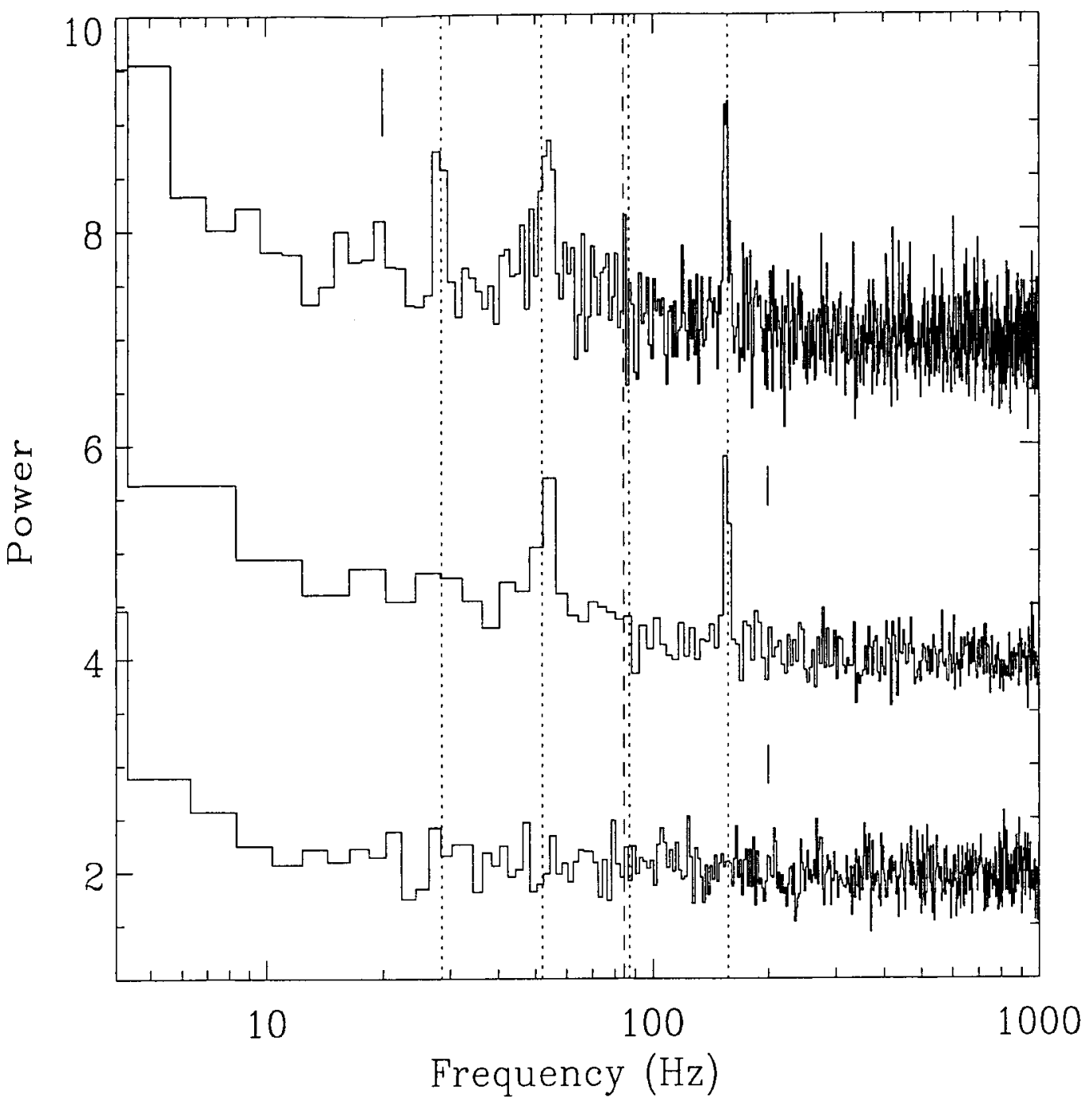

Fig. 4.- Average power spectra from $1.5 \mathrm{~s}$ intervals centered at different rotational phases. The upper curve was computed using 20 successive $1.5 \mathrm{~s}$ intervals centered on the rotational phase of the $84 \mathrm{~Hz}$ QPO (see Fig. 3). The frequency resolution is $4 / 3 \mathrm{~Hz}$. The middle curve shows the same spectrum with $4 \mathrm{~Hz}$ resolution. Two QPOs are clearly detected in this spectrum, and a third, weaker feature is present at the higher frequency resolution (top, see the text for details). The lower curve is from reference phases $\pm 1.5 \mathrm{~s}$ away from the $84 \mathrm{~Hz}$ signal phase. No QPOs are detected at these phases. The vertical dotted lines mark the frequencies of $l=2,4,7$, and $13{ }_{l} t_{0}$ modes, in order of increasing frequency, assuming that the ${ }_{2} t_{0}$ mode has a frequency of $28.5 \mathrm{~Hz}$. The vertical dashed line marks the frequency of the transient $84 \mathrm{~Hz}$ QPO. At the $5.16 \mathrm{~s}$ period, $1.5 \mathrm{~s}$ corresponds to 0.3 cycles, or about $105^{\circ}$. Characteristic error bars are shown for each spectrum. 


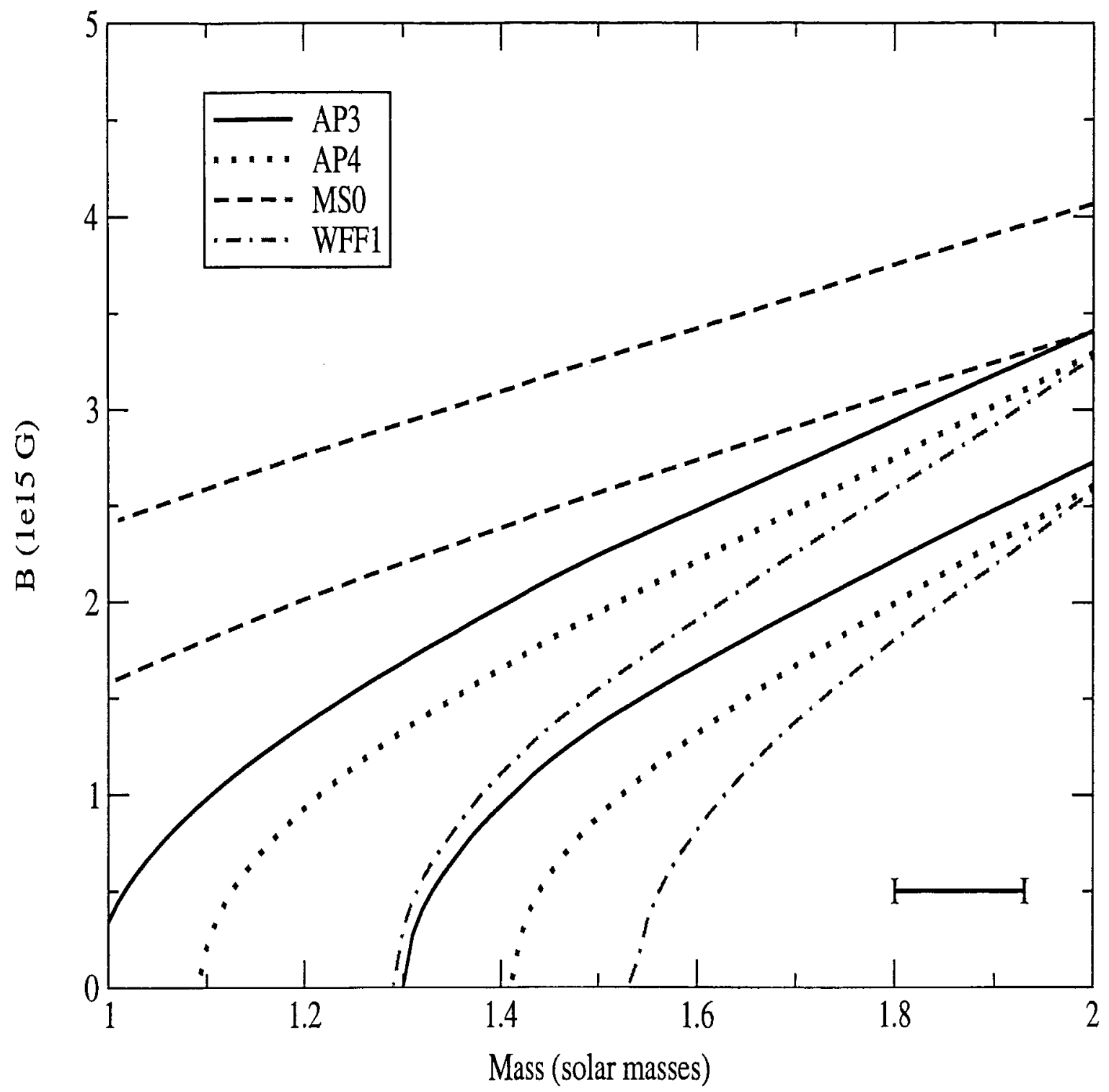

Fig. 5.- The stellar mass and magnetic field required to give the ${ }_{2} t_{0}$ mode frequencies inferred for $1806(30.4 \mathrm{~Hz})$ and $1900(\approx 28 \mathrm{~Hz})$. We model four of the EOS discussed in Lattimer \& Prakash (2001); in order of increasing stiffness they are WFF1, AP4, AP3 and MS0. For each EOS two lines are shown. The upper line indicates the parameters necessary to give $30.4 \mathrm{~Hz}$, the lower line $28 \mathrm{~Hz}$. The horizontal line indicates the uncertainty in the position of the footprint of the 1900 AP3, AP4 and WFF1 lines due to the $\pm 0.5 \mathrm{~Hz}$ uncertainty on the $28 \mathrm{~Hz}$ frequency. 